\title{
CALCULATION OF LOW MACH NUMBER ACOUSTICS: A COMPARISON OF MPV, EIF AND LINEARIZED EULER EQUATIONS
}

\author{
Sabine Roller $^{1}$, Thomas SchwartzkopfF ${ }^{2}$, Roland Fortenbach $^{2}$, \\ Michael Dumbser ${ }^{2}$ and Claus-Dieter Munz ${ }^{2}$
}

\begin{abstract}
The calculation of sound generation and propagation in low Mach number flows requires serious reflections on the characteristics of the underlying equations. Although the compressible Euler/Navier-Stokes equations cover all effects, an approximation via standard compressible solvers does not have the ability to represent acoustic waves correctly. Therefore, different methods have been developed to deal with the problem. In this paper, three of them are considered and compared to each other. They are the Multiple Pressure Variables Approach (MPV), the Expansion about Incompressible Flow (EIF) and a coupling method via heterogeneous domain decomposition. In the latter approach, the non-linear Euler equations are used in a domain as small as possible to cover the sound generation, and the locally linearized Euler equations approximated with a high-order scheme are used in a second domain to deal with the sound propagation. Comparisons will be given in construction principles as well as implementational effort and computational costs on actual numerical examples.
\end{abstract}

Mathematics Subject Classification. 41A60, 65M55, 76Q05.

Contributed talk, Low Mach Number Flows Conference, June 21-25, 2004, Porquerolles, France.

\section{INTRODUCTION}

During the last decades, aero-acoustic simulations became more and more important in many technical applications. Not only in aircraft industry, where the noise generated at take-off and landing of air planes is an important impact factor on the landing fees of the airports, but also in automotive and computer industry, even in housing and buildings where the loudness of the air condition becomes a selling argument.

Therefore, generation and propagation of sound waves in fluid flow become an important aspect in numerical simulations. Acoustic waves are weakly compressible phenomena, especially pressure and density fluctuations, in an besides this incompressible flow. Therefore, acoustic waves are covered by the compressible Navier-Stokes equations which are well known equations with a large variety of numerical simulation schemes. Unfortunately, the devil is in the details. Although the governing equations are well known and treated numerically for years, the simultaneous calculation of the flow generating the noise and the sound waves spreading from this source for low Mach numbers is still an active field of research. The key difficulty is the large discrepancy between a) the two occurring speeds, namely the flow speed on the one hand and the speed of sound on the other; b) the amount

Keywords and phrases. Aero-acoustics, low Mach number flows, asymptotic expansion, heterogeneous domain decomposition.

${ }^{1}$ High Performance Computing Center Stuttgart (HLRS), University of Stuttgart, Germany. roller@hlrs.de

2 Institute for Aerodynamics and Gasdynamics (IAG), University of Stuttgart, Germany. schwartzkopff@iag.uni-stuttgart.de

(c) EDP Sciences, SMAI 2005 
of energy, i.e. the amplitude of the pressure waves which are related to the flow itself and the sound waves respectively; and c) the length scales on which the effects take place, i.e. the extent of the domain of interest as well as the necessary resolution in space and/or time.

This paper is divided in the following sections: first, we will shortly review an asymptotic analysis to get insight into the behavior of a nearly incompressible fluid flow. The knowledge gained from this analysis will then be used in the second section to construct the so called MPV scheme, a numerical scheme well suited for low Mach number flow calculations. The third section will review another ansatz which is motivated by the asymptotic expansion in the first section, i.e. the Expansion about Incompressible Flow (EIF). Here, the flow is assumed to be an incompressible flow with perturbations and evolution equations for the perturbations will be derived. The fourth section describes yet another access to the problem, that is the use of the full compressible equations without any assumptions or modeling by just using arbitrarily high order schemes. However, to reduce the computational effort the equations are used in their original non-linear form only in a domain as small as possible to cover the sound generation, while in the far field the linearized equations will be used. Thus, the coupling mechanism will be described in the fifth section. The sixth section will describe numerical results and comparisons between the three presented schemes. The paper closes with concluding remarks.

\section{Asymptotic AnAlysis for LOW Mach NUmber FlOWs}

The asymptotic considerations in this paper are based on the compressible Navier-Stokes equations. In conservative formulation they read as

$$
\begin{aligned}
\rho_{t}+\nabla \cdot(\rho \mathbf{U}) & =0 \\
(\rho \mathbf{U})_{t}+\nabla \cdot((\rho \mathbf{U}) \circ \mathbf{U})+\nabla p & =\nabla \cdot \tau+\rho \mathbf{g}, \\
e_{t}+\nabla \cdot(\mathbf{U}(e+p)) & =\nabla \cdot(\tau \mathbf{U})+\rho \mathbf{g} \cdot \mathbf{U}-\nabla \cdot \mathbf{q},
\end{aligned}
$$

where $\rho$ denotes the density, $\mathbf{U}$ the velocity vector, $p$ the total pressure, and $e$ the total energy per unit volume. The stress tensor $\tau$ is given by

$$
\tau=2 \mu \mathbf{D}-\frac{2}{3} \mu \nabla \mathbf{U} \quad \text { with } \quad \mathbf{D}=\frac{1}{2}\left[\nabla \mathbf{U}+(\nabla \mathbf{U})^{T}\right],
$$

the heat flux $q$ is proportional to the gradient of the temperature $T$,

$$
\mathbf{q}=-\lambda \nabla T \text {. }
$$

The total energy consists of specific internal energy $\varepsilon$, the kinetic energy and the potential energy,

$$
e=\rho \varepsilon+\frac{1}{2} \rho \mathbf{U}^{2}+\rho g h .
$$

The system is closed by an equation of state which links the pressure to density and internal energy. The equation of state of an ideal gas is

$$
p=(\gamma-1) \rho \varepsilon .
$$

In this paper, the governing equations are often used in a formulation in primitive variables $(\rho, \mathbf{U}, p)$ instead of the conservative variables $(\rho, \rho \mathbf{U}, e)$ since the connection to incompressible equations is more obvious in this 
formulation. In non-dimensional form they are given as

$$
\begin{gathered}
\rho_{t}+\mathbf{U} \cdot \nabla \rho+\rho \nabla \cdot \mathbf{U}=0 \\
\mathbf{U}_{t}+(\mathbf{U} \cdot \nabla) \mathbf{U}+\frac{1}{\mathrm{M}^{2} \rho} \nabla p=\frac{1}{\operatorname{Re} \rho} \Delta \mathbf{U}+\frac{1}{3 \operatorname{Re} \rho} \nabla(\nabla \cdot \mathbf{U})+\frac{1}{\operatorname{Fr}^{2}} \mathbf{g} \\
p_{t}+\mathbf{U} \cdot \nabla p+\gamma p \nabla \cdot \mathbf{U}=\frac{\gamma}{\operatorname{PrRe}} \Delta T .
\end{gathered}
$$

The non-dimensional quantities in (6) are the Mach number $\mathrm{M}=u_{\text {ref }} / \sqrt{p_{\text {ref }} / \rho_{\text {ref }}}$, the Reynolds number $\operatorname{Re}=x_{\text {ref }} \rho_{\text {ref }} u_{\text {ref }} / \mu$, the Prandtl number $\operatorname{Pr}=\mu c_{p} / \lambda$ and the Froude number $\operatorname{Fr}=\sqrt{u_{\text {ref }}^{2} / g_{\text {ref }} x_{\text {ref }}}$. Defined in that way, all four quantities are global values. Their local values can differ from that.

The Mach number was introduced by choosing appropriate reference values $u_{\text {ref }}$ for the flow velocity and $c_{\text {ref }}=\sqrt{p_{\text {ref }} / \rho_{\text {ref }}}$ for the speed of sound, respectively. In the fully compressible flow regime they are chosen equally, $u_{\text {ref }}=c_{\text {ref }}$, i.e. $p_{\text {ref }}=\rho_{\text {ref }} u_{\text {ref }}^{2}$. In the case of essentially incompressible flows, this choice is inappropriate since the flow velocity is by some orders of magnitude smaller than the speed of sound. Therefore, the reference value for the speed of sound is chosen independently of the reference value for the flow velocity by choosing some suitable reference value $p_{\text {ref }}, e . g . p_{\text {ref }}=p_{\text {mean }}$ as the mean pressure or $p_{\text {ref }}=p_{\infty}$ as the constant pressure in the far field. By this choice, the Mach number occurs as a parameter, characterizing the ratio of flow speed to speed of sound in the flow of interest, i.e. it is a measure for the compressibility of the flow.

Since the Mach number occurs as parameter in the equations, the flow variables density, velocity and pressure depend on the Mach number as well. Therefore, an asymptotic expansion of the form

$$
f(\mathbf{x}, t ; \mathrm{M})=f^{(0)}(g(\mathbf{x}, t, \mathrm{M}))+\mathrm{M} f^{(1)}(g(\mathbf{x}, t, \mathrm{M}))+\mathrm{M}^{2} f^{(2)}(g(\mathbf{x}, t, \mathrm{M}))+o\left(\mathrm{M}^{2}\right)
$$

is chosen with $f$ denoting any of the flow variables mentioned. If $g(\mathbf{x}, t, \mathbf{M})=(\mathbf{x}, t)$, then the expansion is called a single scale expansion since it uses only one single scale in space as well as in time. In this case, the expansion functions $f^{n}$ are independent of the Mach number. An asymptotic analysis of this form was investigated by Klainerman and Majda [5]. They could show that in the limit when M tends to zero, the solution of the compressible equations converges to the solution of the incompressible equations.

This form of asymptotic expansion represents effects, which in the limit $\mathrm{M} \rightarrow 0$ differ strongly in their amplitudes. In the case of low Mach number aero-acoustics, also phenomena have to be covered which differ for $\mathrm{M} \rightarrow 0$ not only in their amplitudes but also in the characteristic length scales on which they act. The flow itself carries a large amount of energy and develops on small length scales representing vortices, for example. The acoustic waves generated by the deformation of the vortices on the other hand carry only small amounts of energy, but travel over large spatial distances. To cover these different length scales also, a transformation $g$, depending on the Mach number, is introduced which transforms one or both of the independent variables $\mathbf{x}$ and $t$ onto several variables. In this paper, a single time scale, two space scales expansion is used, $i . e$. $g(\mathbf{x}, t ; \mathrm{M})=(x, \xi=\mathrm{Mx}, t)$. With this transformation, the spatial derivatives become $\nabla f=\nabla_{x} f+\mathrm{M} \nabla_{\xi} f$. This kind of expansion was introduced by Klein [6] and Meister [8].

Introducing this expansion into the equations (6) and balancing all terms of the same power of the Mach number, the asymptotic equations of leading, first and second order are obtained [11]. Omitting the details here, the results of the asymptotic analysis can be summarized as follows:

- The usual hope by applying an asymptotic analysis is to obtain a closed system of equations of leading order which can be solved more easily then the original ones but resulting in a good approximation to the solution of the original equations. The first order equations than form again a closed system of equations (more or less) easy to solve, containing the results of the leading order system as source terms on the right hand side. The solution of the first order system improves then the approximation obtained. Applying the second order system, the solution again is improved and this algorithm can be applied up to a solution of a certain accuracy. 
Unfortunately, this hope fails for the Euler and Navier-Stokes equations. It is not possible to obtain a closed system for the leading order terms. Neither it is true for the first order nor the second order terms. The smallest closed system that can be obtained from the asymptotic analysis is a system containing the leading order density, leading order velocity and leading as well as second order pressure terms. This indicates, that the pressure has to play at least two different roles in the equations which in the limit cannot be expressed in one variable.

- The different roles of the pressure can be interpreted mathematically as well as physically. The leading order pressure term $p^{(0)}$ acts as a thermodynamic quantity. It fulfills the equation of state and becomes constant in the zero-Mach number limit. In this case, it represents the constant background pressure.

- The first order pressure $p^{(1)}$ acts as acoustic variable. It fulfills linear acoustic equations on the large space scale $\xi$, but is constant on the small flow scale $x$. Therefore, in low Mach number flows without acoustic phenomena, it can be neglected, while in the presence of acoustic waves it represents large scale, long wave length effects of small amplitude.

- The second order pressure term $p^{(2)}$ acts as a Lagrangian multiplier or balance-of-forces-agent in the zeroMach number limit. It guarantees for the divergence constraint on the velocity which requires divergence free velocities in the incompressible limit without compression from the outside or constant divergence velocities in the case of compression or heating from the boundaries. This divergence constraint is defined by the background pressure $p^{(0)}$, but fulfilled by the second order pressure $p^{(2)}$ which is therefore called the incompressible pressure.

- The higher order terms of density and velocity in contrast influence only other higher order terms and can therefore be neglected in the construction of a numerical approximation scheme.

In summary, equations (6) converge in the the limit $\mathrm{M} \rightarrow 0$ to the equations

$$
\begin{gathered}
\rho_{t}^{(0)}+\mathbf{U}^{(0)} \cdot \nabla_{x} \rho^{(0)}+\rho^{(0)} \nabla_{x} \cdot \mathbf{U}^{(0)}=0 \\
\mathbf{U}_{t}^{(0)}+\left(\mathbf{U}^{(0)} \cdot \nabla_{x}\right) \mathbf{U}^{(0)}+\frac{1}{\rho^{(0)}} \nabla_{x} p^{(2)}=\frac{1}{\operatorname{Re} \rho^{(0)}} \Delta_{x x} \mathbf{U}^{(0)}+\frac{1}{\operatorname{Fr}^{2}} \mathbf{g} \\
\nabla_{x} \cdot \mathbf{U}^{(0)}=-\frac{p_{t}^{(0)}}{\gamma p^{(0)}} \\
p_{t}^{(0)}=-\frac{\gamma p^{(0)}}{|V|} \int_{\partial V} \mathbf{U}^{(0)} \cdot \mathbf{n} \mathrm{d} s .
\end{gathered}
$$

This is the system of incompressible Navier-Stokes equations with variable density and possible compression from the boundaries such as a piston movement. Without compression from the outside, $p_{t}^{(0)}$ becomes zero, the right hand side of equation (8c) vanishes and the velocity becomes divergence free. Then, the continuity equation (8a) becomes a pure transport equation for any initially given density distribution.

To obtain this limit system, heat transfer was neglected. This was done only to make the convergence to the incompressible equations in their usual form more obvious. In principle, heat transfer in nearly incompressible flow regimes can be treated by these equations as well. This is important for thermo-acoustical phenomena which are not subject of this paper. The acoustic scale $\xi=\mathrm{M} x$ in general vanishes in the limit $\mathrm{M} \rightarrow 0$ such that the acoustic pressure term becomes void without any additional assumptions.

\section{The Multiple Pressure Variables (MPV) scheme}

Since the asymptotic equations are valid only in the limited range of very small Mach numbers, the numerical scheme we construct here, is not based on the asymptotic equations but on the full compressible Navier-Stokes equations (6). Three different pressure variables $p^{(0)}, p^{(1)}$ and $p^{(2)}$ are introduced, linked to the total pressure $p$ via the consistency constraint $p=p^{(0)}+\mathrm{M} p^{(1)}+\mathrm{M}^{2} p^{(2)}$. The system of the Navier-Stokes equations has then to be augmented by two additional equations to close the system. These additional equations are the asymptotic 
equations obtained from the above analysis for the leading and first order pressure terms. Then, the pressure $p^{(2)}$ can be interpreted as a rescaling of the total pressure $p$ using the already known values for $p^{(0)}$ and $p^{(1)}$. Thus, the asymptotic equations for these terms are used as a predictor, while errors - especially those occurring in the regime of Mach numbers larger than allowed for the asymptotic analysis - are corrected by using the full compressible equations. Therefore the range of validity for such a system is not restricted to very low Mach numbers only, but in principle allows for the complete subsonic range of Mach numbers. If a suitable numerical scheme is chosen, even trans- and supersonic flows can be calculated but the effort then is not justified.

To guarantee the numerical consistency of the total pressure with the sum of the individual pressure terms, in every time step, the total pressure has to be decomposed into its contributions, their temporal evolution within this time step has to be calculated and the new pressure terms have to be added up again. The decomposition uses the fact, that in the incompressible limit, $p^{(0)}$ becomes constant in space and $p^{(1)}$ constant on the small flow scale, but varies on the large acoustical scale. Therefore, $p^{(0)}$ and $p^{(1)}$ are defined to be the mean value of the total pressure over the whole computational domain $V$ and an acoustic domain $V_{\text {ac }}$, respectively,

$$
p^{(0)}:=\frac{1}{|V|} \int_{V} p \mathrm{~d} x, \quad \quad p^{(1)}:=\frac{1}{\mathrm{M}\left|V_{\mathrm{ac}}\right|} \int_{V_{\mathrm{ac}}} p-p^{(0)} \mathrm{d} x .
$$

In the numerical scheme, for each grid point $V_{\mathrm{ac}}$ is defined as the ball of diameter $x_{\mathrm{ref}} / \mathrm{M}$ around the point since the acoustic scale is by the factor $1 / \mathrm{M}$ larger than the flow scale. The characteristic length scale $x_{\text {ref }}$ is chosen as an characteristic resolution for the flow scale, usually as $\Delta x$ on an equidistant or some mean value of grid cell length' on a non-equidistant mesh. The pressure $p^{(2)}$ is then defined for consistency as

$$
p^{(2)}:=\frac{1}{\mathrm{M}^{2}}\left(p-p^{(0)}-\mathrm{M} p^{(1)}\right) .
$$

The temporal evolution of $p^{(0)}$ and $p^{(1)}$ are then calculated via the asymptotic equations. The leading order pressure $p^{(0)}$ is determined completely by compression or heat transfer from the boundaries,

$$
p_{t}^{(0)}=-\frac{\gamma p^{(0)}}{|V|} \int_{\partial V} \mathbf{U} \cdot \mathbf{n} \mathrm{d} s+\frac{\gamma}{\operatorname{PrRe}|V|} \int_{\partial V} \nabla_{x} T \cdot \mathbf{n} \mathrm{d} s .
$$

Without compression or heat transfer from the outside, it becomes constant in time as well.

The change of $p^{(1)}$ within one time step is obtained by solving the linear acoustical system

$$
\begin{aligned}
p_{t}^{(1)}+\gamma p^{(0)} \nabla_{\xi} \overline{\mathbf{U}} & =0, \\
\overline{\mathbf{U}}_{t}+\frac{1}{\bar{\rho}} \nabla_{\xi} p^{(1)} & =-\frac{\overline{(\tilde{\rho} \tilde{\mathbf{U}})_{t}}}{\bar{\rho}}
\end{aligned}
$$

which was obtained from the asymptotic analysis. The terms $\bar{\rho}$ and $\overline{\mathbf{U}}$ represent the long wavelength contributions of density and velocity and are defined as

$$
\bar{\rho}:=\frac{1}{\left|V_{\mathrm{ac}}\right|} \int_{V_{\mathrm{ac}}} \rho \mathrm{d} x, \quad \overline{\mathbf{U}}:=\frac{1}{\left|V_{\mathrm{ac}}\right|} \int_{V_{\mathrm{ac}}} \mathbf{U ~ d} x .
$$

This completes the predictor step. The equations solved by the Multiple Pressure Variables (MPV) scheme, given in primitive variables, are then

$$
\begin{gathered}
\rho_{t}+\mathbf{U} \cdot \nabla \rho+\rho \nabla \cdot \mathbf{U}=0, \\
\mathbf{U}_{t}+(\mathbf{U} \cdot \nabla) \mathbf{U}+\frac{1}{\rho} \nabla p^{(2)}=-\frac{1}{\mathrm{M} \rho} \nabla p^{(1)}+\frac{1}{\operatorname{Re} \rho} \Delta \mathbf{U}+\frac{1}{3 \operatorname{Re} \rho} \nabla(\nabla \cdot \mathbf{U})+\frac{1}{\operatorname{Fr}^{2}} \mathbf{g}, \\
\mathrm{M}^{2} p_{t}^{(2)}+\mathrm{M}^{2} \mathbf{U} \cdot \nabla p^{(2)}+\mathrm{M}^{2} \gamma p^{(2)} \nabla \cdot \mathbf{U}=-p_{t}^{(0)}-\mathrm{M} p_{t}^{(1)}-\mathrm{M} \mathbf{U} \cdot \nabla p^{(1)}-\gamma\left(p^{(0)}+\mathrm{M} p^{(1)}\right) \nabla \cdot \mathbf{U}+\frac{\gamma}{\operatorname{Pr} \operatorname{Re}} \Delta T .
\end{gathered}
$$




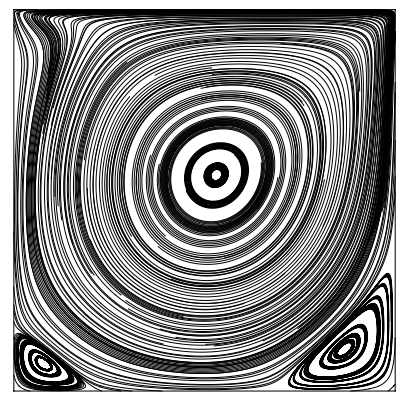

FIGURE 1. Streamline visualization of the lid driven cavity flow at $\operatorname{Re}=1000$.

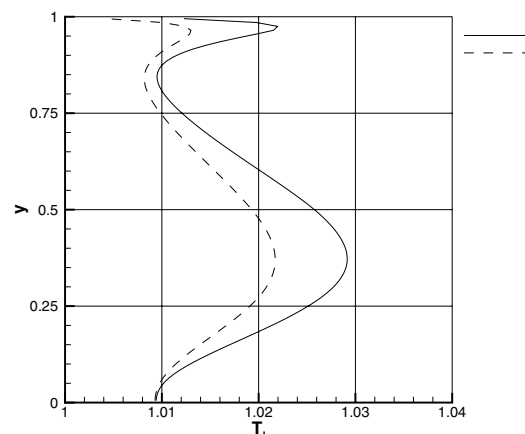

Figure 2. Temperatures at the left (cold) walls.

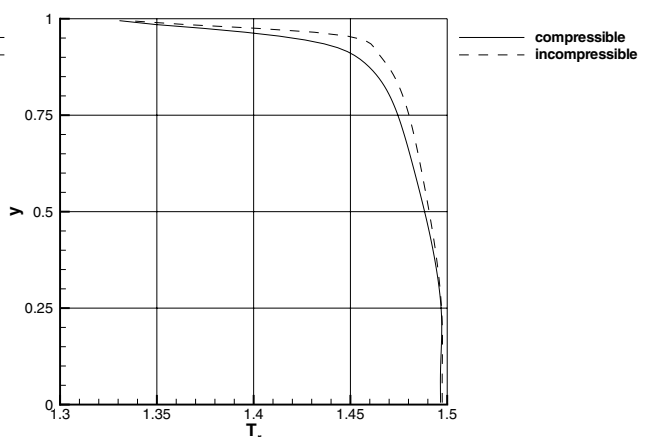

Figure 3. Temperatures at the right (hot) walls.

Any numerical scheme can be applied to these equations. The advantage of the MPV algorithm as described above is that it results in an all Mach number code which can be formulated in such a way, that it converges to a standard incompressible scheme in the limit $\mathrm{M} \rightarrow 0[7,10-12]$ or to an standard Godunov scheme for $\mathrm{M} \rightarrow 1[3,13]$. It also allows for a re-coupling of the acoustic phenomena to the flow field. This is important for thermo-acoustic applications in closed reaction chambers where the sound waves extract energy in one region of the flow and feed it back in another. This can determine for example whether flames extinct or not in some regions of the flow.

The computations shown in this paper have been realized by splitting the equations into a convection-diffusion system which is solved explicitly for the convection terms, implicitly for the diffusion terms. The CFL-condition for the explicit time step restriction then includes the flow velocity only, therefore appropriate time steps can be chosen even for very low Mach numbers. The second part of the splitting scheme treats all the sonic terms which is therefore done implicitly to avoid tiny time steps. All discretizations have been done by finite difference schemes second order in time and space. The grid used here was a staggered non-equidistant Cartesian grid.

The discretization of the acoustic system (12) includes the discretization of the large scale derivatives $\nabla_{\xi}$. This has been done by restricting the flow variables $\rho, \mathbf{U}$ and $p^{(1)}$ to a much coarser grid with a coarsening factor of $1 / \mathrm{M}$. This can be viewed as a physically motivated multi-grid algorithm. The computational effort for acoustic calculations is reduced by this since on the coarse grid the Mach number cancels out of the CFL-condition for the acoustic system and a time step of the same order of magnitude as for the convection step can be chosen.

We computed the well-known lid driven cavity at $\mathrm{Re}=1000$ as an incompressible example extended to the weakly compressible regime by differentially heating the vertical walls. The left wall was kept at a constant temperature of $20{ }^{\circ} \mathrm{C}(293.6 \mathrm{~K})$. The right wall was heated to $293.6 \mathrm{~K}+\Delta T$. As long as the temperature difference $\Delta T$ is small, the density can be assumed to be constant and the incompressible approximation can be applied. For $\Delta T$ larger than a few degrees, this assumption is no longer valid. To show this, we applied a temperature difference $\Delta T=150 \mathrm{~K}$ and compared the results of the MPV scheme with those obtained by an incompressible approximation plus temperature equation. The Mach number for this test case is $\mathrm{M}=0.0005$, the Prandtl number $\operatorname{Pr}=0.7$ and the Reynolds number $\operatorname{Re}=1000$ as above. Gravity is not considered in this example, the driving force is the movement of the upper wall. Figure 1 shows the streamlines which represent a large vortex in the center of the cavity and two smaller ones in the lower left and right corners. Figures 2 and 3 show the temperature profiles at the left and right walls.

The solid lines give the results of the MPV scheme, the dashed lines are the results obtained using an incompressible solver with additional temperature equation. It can be clearly seen, that the incompressible solution shows a thicker thermal boundary layer than the MPV scheme. This is due to the fact that the incompressible calculation cannot reproduce the thermal density changes. The relative $L_{2}$ deviation of the compressible and incompressible results at the left and right wall is listed in Table 1. 
TABLE 1. Relative $L_{2}$ error of density at left and right wall at various temperature differences.

\begin{tabular}{|c|c|c|c|c|c|}
\hline$\Delta T$ & $30 \mathrm{~K}$ & $60 \mathrm{~K}$ & $90 \mathrm{~K}$ & $120 \mathrm{~K}$ & $150 \mathrm{~K}$ \\
\hline$\Delta \rho_{\text {left }}$ & $4.4 \%$ & $8.3 \%$ & $12 \%$ & $15 \%$ & $18 \%$ \\
\hline$\Delta \rho_{\text {right }}$ & $4 \%$ & $7.6 \%$ & $11 \%$ & $14 \%$ & $16 \%$ \\
\hline
\end{tabular}

To show the ability of the MPV scheme to predict the interaction of long wave length acoustic waves with small local entropy fluctuations, we considered baroclinic vortex formation as a test case, the so-called KelvinHelmholtz instability. The initial data, given as follows,

$$
\begin{aligned}
& \rho(x, y, 0)=1.0+0.2 \mathrm{M}(1.0+\cos (\pi x / L))+\Phi(y), \\
& p(x, y, 0)=1.0+\mathrm{M} \gamma(1.0+\cos (\pi x / L)), \\
& u(x, y, 0)=\sqrt{\gamma}(1.0+\cos (\pi x / L)), \\
& v(x, y, 0)=0.0
\end{aligned}
$$

with

$$
\Phi(y)= \begin{cases}\frac{0.8}{L_{y}} y & \text { for } 0 \leq y \leq \frac{1}{2} L_{y}, \\ \left.\frac{0.8}{L_{y}}\left(y-\frac{1}{2} L_{y}\right)\right)-0.4 & \text { for } \frac{1}{2} L_{y} \leq y \leq L_{y},\end{cases}
$$

represent a saw-tooth like density stratification in the vertical direction set into motion by a right-running acoustic pulse in the horizontal direction. At $\mathrm{M}=0.05$, the computational domain is a double-periodic domain of $[-L, L] \times\left[0, L_{y}\right]$ with $L=\frac{1}{\mathrm{M}}=20$ and $L_{y}=\frac{2 L}{5}=8$, just long enough to let one period of the acoustic wave take place. Due to the non-homogeneous density, the fluid is subjected to a higher acceleration for lower density values and a lower acceleration at higher densities. This leads to the well known phenomenon of a Kelvin-Helmholtz instability. A shear layer of sinusoidal shape is generated and moved with the acoustic wave. Figures 4 and 5 show the density and vorticity contours at different times. The first plot shows the initial data, the second one the first forming of the sinusoidal shape at time $t=6$. The interface starts rolling up and small vortices are formed. This can be clearly seen at the later times $t=9,11,14$. The smallest structures resolved have a thickness of a few grid zones length, smaller ones are damped out by numerical viscosity.

\section{Expansion about InCOMPRESSible Flow (EIF)}

For flow situations where no feedback of the acoustics to the flow simulation is necessary, another approach was developed by Hardin and Pope [4], the so called Expansion about Incompressible Flow (EIF). Here, the incompressible equations are solved by any standard incompressible solver. In the solution obtained from that, no acoustic waves are included, but Klainerman and Majda [5] showed that acoustics form a first order correction to these equations. Therefore, in a second step the solution of the compressible equations is expressed as the incompressible solution plus some disturbance terms which represent the acoustics.

To develop the disturbance equations, we denote with $\mathbf{U}_{0}, p_{0}^{(2)}, p_{0}$, and $\rho_{0}$ the velocity, pressure, background pressure and density satisfying the incompressible isentropic equations,

$$
\begin{gathered}
\mathbf{U}_{0, t}+\left(\mathbf{U}_{0} \circ \nabla\right) \mathbf{U}_{0}+\frac{1}{\rho_{0}} \nabla p^{(2)}=0, \\
\nabla \cdot \mathbf{U}_{0}=0, \\
p_{0} \equiv \text { const., } \rho_{0}=p_{0}^{1 / \gamma},
\end{gathered}
$$




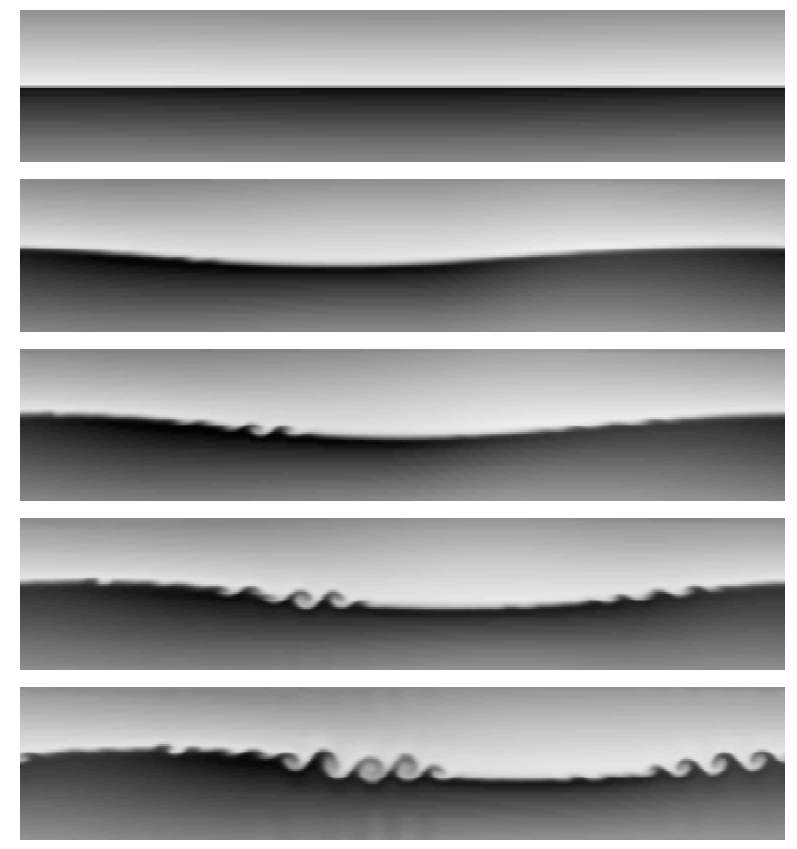

Figure 4 . Density profile at times $t=0,6,9$, 11,14 .
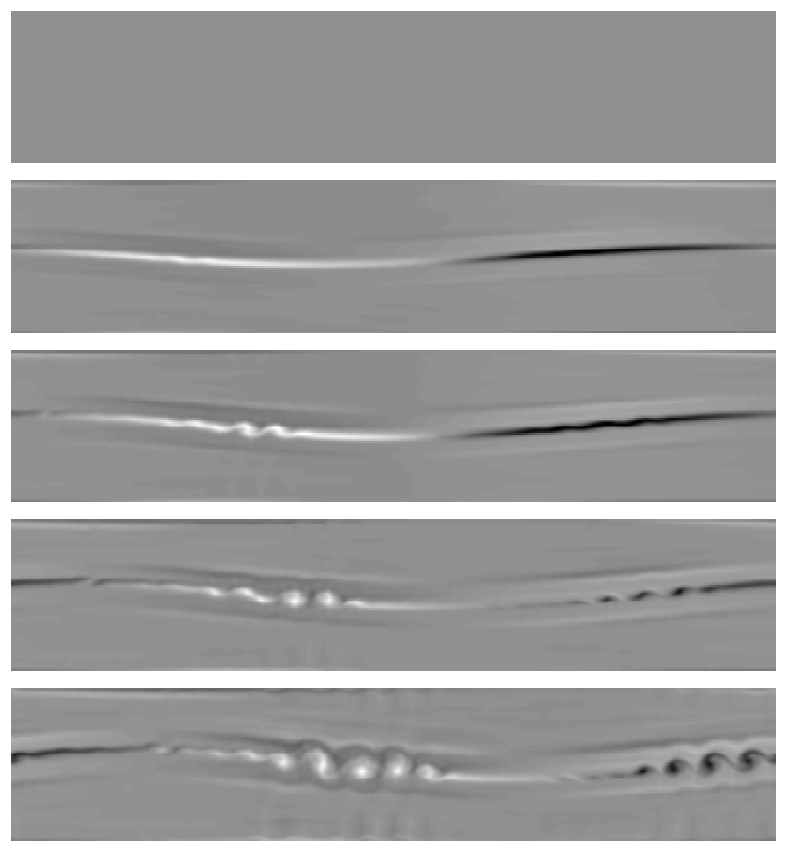

FiguRE 5. Vorticity profile at times $t=0,6,9$, 11,14 .

and with $\rho^{\prime}$, $\mathbf{U}^{\prime}$, and $p^{\prime}$ the perturbation functions that capture compressible effects. As an extension of the original EIF approach, the perturbations are scaled with the Mach number here, see [2]. Then, a scaled perturbation expansion is set as

$$
\begin{aligned}
& \rho=\rho_{0}+\mathrm{M}^{2} \rho^{(2)}+\mathrm{M}^{2} \rho^{\prime}, \\
& \mathbf{U}=\mathbf{U}_{0}+\mathbf{M U}^{\prime}, \\
& p=p_{0}+\mathrm{M}^{2} p^{(2)}+\mathrm{M}^{2} p^{\prime} .
\end{aligned}
$$

The expansion (15) is introduced into the compressible Euler equations and higher order terms are neglected. As the incompressible solution is already known, we end up with the system for the perturbation functions as

$$
\begin{aligned}
& \rho_{t}^{\prime}+\mathbf{U}_{0} \cdot \nabla \rho^{\prime}+\frac{\rho_{0}}{\mathrm{M}} \nabla \cdot \mathbf{U}^{\prime}=-\rho_{t}^{(2)}-\mathbf{U}_{0} \cdot \nabla \rho^{(2)}, \\
& \mathbf{U}_{t}^{\prime}+\left(\mathbf{U}_{0} \circ \nabla\right) \mathbf{U}^{\prime}+\left(\mathbf{U}^{\prime} \circ \nabla\right) \mathbf{U}_{0}+\frac{1}{\mathrm{M} \rho_{0}} \nabla p^{\prime}=0, \\
& p_{t}^{\prime}+\mathbf{U}_{0} \cdot \nabla p^{\prime}+\frac{\gamma p_{0}}{\mathrm{M}} \nabla \cdot \mathbf{U}^{\prime}=-p_{t}^{(2)}-\mathbf{U}_{0} \cdot \nabla p^{(2)} .
\end{aligned}
$$

Assuming additionally the incompressible flow to be zero in the far field (i.e. $\mathbf{U}_{0}=0, p^{(2)} \equiv$ const.), then system (16) reduces to the classical wave equation in the far field

$$
\begin{aligned}
& \rho_{t}^{\prime}+\frac{\rho_{0}}{\mathrm{M}} \nabla \cdot \mathbf{U}^{\prime}=0, \\
& \mathbf{U}_{t}^{\prime}+\frac{1}{\mathrm{M} \rho_{0}} \nabla p^{\prime}=0, \\
& p_{t}^{\prime}+\frac{\gamma p_{0}}{\mathrm{M}} \nabla \cdot \mathbf{U}^{\prime}=0 .
\end{aligned}
$$




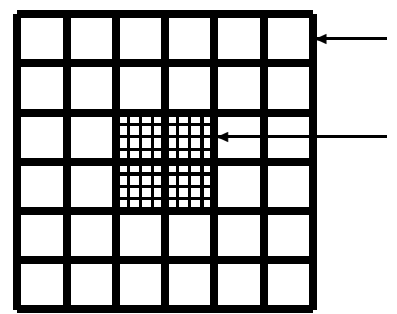

acoustics domain, coarse grid

flow domain (source region), fine grid

Figure 6. Principle of embedded domains.

Thus, the so-called Expansion about Incompressible Flow (EIF) shows how to decouple the sound generation (i.e. the source terms on the right hand side of equations (16) determined by the incompressible solution) from the sound propagation (i.e. the evolution equations for the perturbation functions). Another point to be mentioned here, is that from equations (16) it can be clearly seen that the wavelength of the sound waves is much larger than the characteristic length scale of the acoustic source since the gradient of the pressure is scaled by $1 / \mathrm{M}$.

The advantage of this scheme is a much lower computational and implementational effort. The incompressible equations can be solved by any standard solver which is therefore easily exchangeable. It uses only pressure and velocity, i.e. one variable and therefore one equation less than needed for the compressible equations.

The coupling with the acoustic far field is done via embedded domains, see Figure 6 . The calculation domain consists of a small domain discretized by a fine grid where the incompressible or weakly compressible equations are solved with the MPV-algorithm. Here, slow moving flow phenomena with small vortex and entropy structures require a suitably fine grid. With the results of this calculation, the sound generating source terms for equations (16) can be determined. On top of the flow domain, the acoustic domain is much larger since it has to recover the far field. But due to the fact that only large wavelengths have to be recovered, also a much coarser grid can be applied such that despite the fast sound speed, the same time step as for the flow calculation is acceptable. Nevertheless, high order schemes are necessary to guarantee a good representation of the acoustic waves over a long distance. The algorithm then propagates as follows:

(1) advance the incompressible flow calculation on the fine grid to the next time level;

(2) calculate the acoustic source terms on the fine grid;

(3) restrict the acoustic source terms from the fine grid to the coarse grid;

(4) advance the acoustic calculation on the coarse grid to the next time level.

It is obvious that with this approach acoustic waves cannot influence the flow itself. Therefore it is not applicable to flows e.g. in pipes or burning chambers where interaction is essential. But for far-field sound calculation where interaction between fluid and flow doesn't play an important role it is a fast useful scheme. An open problem unfortunately is that there are different perturbation ansatzes all leading to slightly different source terms for the acoustic equations.

\section{Direct Simulation of Sound generation And PRopagation - Heterogeneous DOMAIN DECOMPOSITION}

Direct simulation of both fluid flow and noise within one numerical calculation offers a valuable means of testing the validity and applicability of aero-acoustic theories and analogies because it does not suffer from uncertainties concerning the modeling of aero-acoustic source terms. But even with today's high performance computers and sophisticated numerical methods this is only possible for relatively simple problems. To reduce computational costs we focus on the domain decomposition approach. 

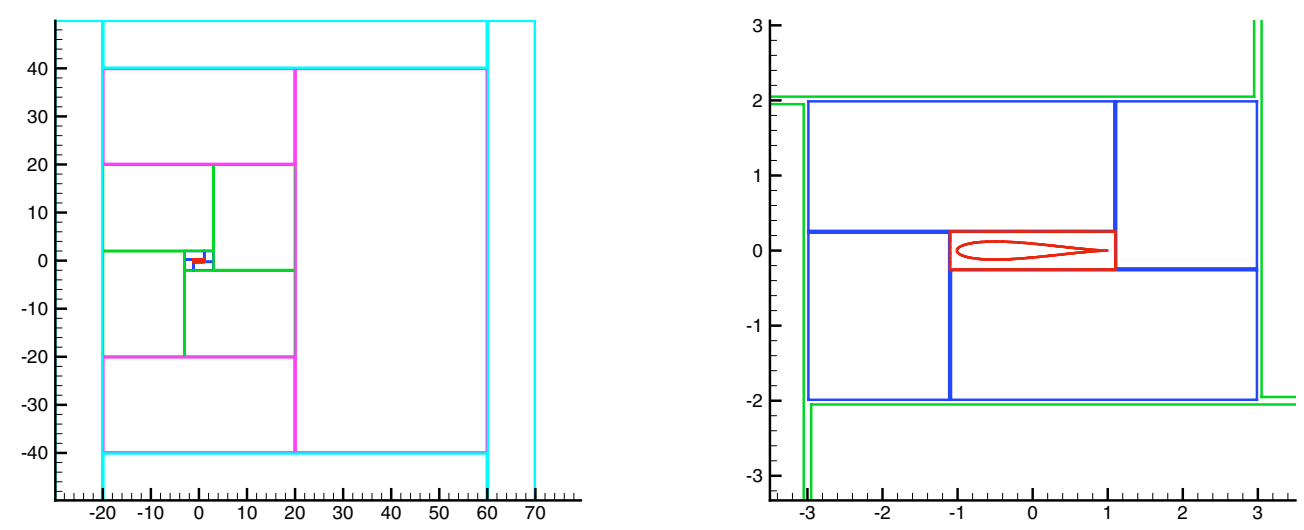

FiguRE 7. Domains used for calculation. Left: overview over all domains, including sponge layer domains. Right: zoom of the core regions, where the nonlinear equations are solved.

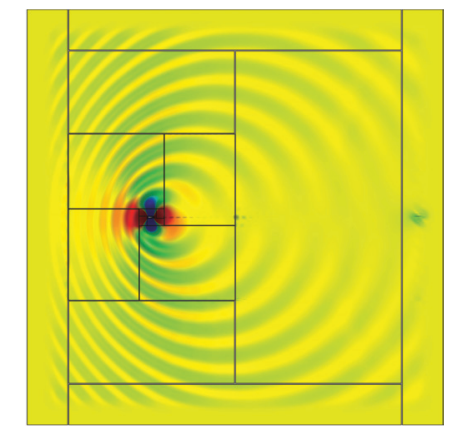

FiguRE 8. Full acoustical + hydrodynamical solution.

In contrast to the approach above where the coupling of the flow domain with the acoustical domain was done over the complete volume by generating the source terms in each cell, the idea now is to use different domains and to adapt each domain to geometrical and physical constraints. The full non-linear Euler or NavierStokes equations are solved on unstructured meshes in the neighborhood of obstacles or other sound generating phenomena. As soon as possible we switch to a structured mesh but still the non-linear equations are used. In the far-field the linearized Euler equations are applied in the regime where nonlinear effects can be neglected. The coupling of the domains is done over the surface only. In this approach only data at the domain interfaces have to be exchanged and no source terms have to be calculated as in the EIF approach. On the other hand we must ensure that the acoustics are well resolved in every domain. An example of such a domain decomposition is given in Figure 7.

Interface conditions must guarantee that all phenomena pass the interface smoothly. The main difficulty here is to avoid non-physical reflections at the artificial interface between different mathematical models, grids and numerical methods. At an interface one has to handle jumps in the following aspects: 1 . a jump in the mesh (different cell sizes, structured-unstructured meshes); 2. a jump in the mathematical modeling (Navier-Stokes, Euler, linearized Euler); 3. different numerical schemes (Finite-Volume, Finite-Difference, Discontinuous-Galerkin) 

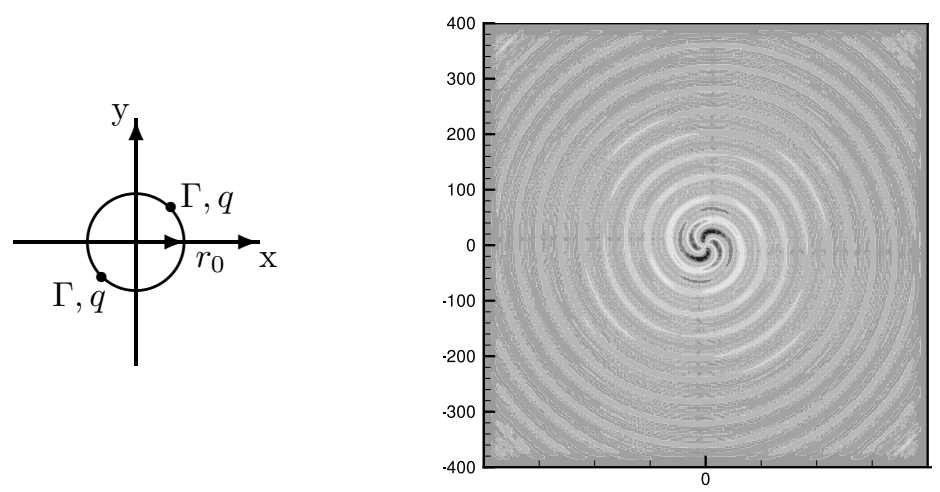

Figure 9. Initial setting and contour plot of the acoustic pressure.

and 4. different time-steps (we use an optimal time-step w.r.t. CFL condition). It can be shown that coupling of different mesh-sizes and time steps can be done such as to ensure globally high order of accuracy [14].

A commonly used idea for these kind of interfaces is to postulate constant fluxes and thus to generate a globally conservative scheme. In our context we have to look a little bit more carefully at the underlying physics. The physical medium, which is modeled by different equations is always one and the same: air. At an interface we have on the one side the Euler equations with the eigenvalues $u \pm c$ and $u$ and on the other side the linearized Euler equations with the linear eigenvalues $u_{0} \pm c_{0}$ and $u_{0}$. Thus the wave speeds are slightly different. If this is interpreted again in a physical context we have two different media with different wave speeds. Thus we get phenomena, which can be predicted by the easy methods of wave physics, such as reflection and diffraction if this interface is modeled exactly in the mathematical context. Clearly these effects are physically wrong in this case as the interface has an arbitrary position and the medium modeled is air everywhere. The values which should be kept constant over the interface are the primitive variables. If this condition is prescribed, the sum

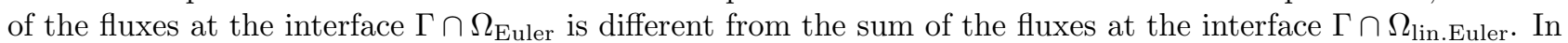
[15], it was shown that this reduces reflections considerably.

For the solution of aero-acoustical problems, it is necessary to use high order schemes to resolve the wave propagation accurately over long distances. Finite-difference schemes are very popular in the aero-acoustic community. They are easy to implement and high spatial order can be achieved easily. For time integration a Runge-Kutta type scheme is used commonly. The problem is that Runge-Kutta integration schemes of an order higher than four get more and more inefficient because the number of stages becomes larger than the formal order of accuracy. A combination of a spatial discretization of order higher than four with Runge-Kutta fourth order time integration remains at fourth order overall accuracy.

For the calculations in this paper, the fastADER scheme was used [18]. The fastADER approach is not suffering from the order restriction of the Runge-Kutta schemes, the so called Butcher barrier. The scheme has uniform arbitrary order in space and time. For the calculation of the time-integrated fluxes at the cell boundaries in the ADER-framework, the solution is expanded in a Taylor series in time where then time-derivatives are replaced by spatial derivatives by successive use of the original PDE. This is the so-called Lax-Wendroff or Cauchy-Kowalewski procedure. A Generalized Riemann Problem is solved to define the interface values of the state and the space-derivatives. The method was first developed in a Finite Volume (FV) context by Toro $[19,20]$. The fast ADER formulation is a reformulation of the ADER approach, in such a way that the kernel of the schemes becomes only a sum over matrix-vector multiplications, which can be optimized highly efficiently. However, if properties of a Finite-Volume scheme are needed, such as the use of Riemann problem solutions in the presence of strong gradients, it can be switched back to the original formulation without any problems. The schemes can be implemented highly efficiently for globally linearized hyperbolic PDE's discretized on Cartesian grids. The CPU time grows quadratically with the order while no extra memory is needed. On structured grids 

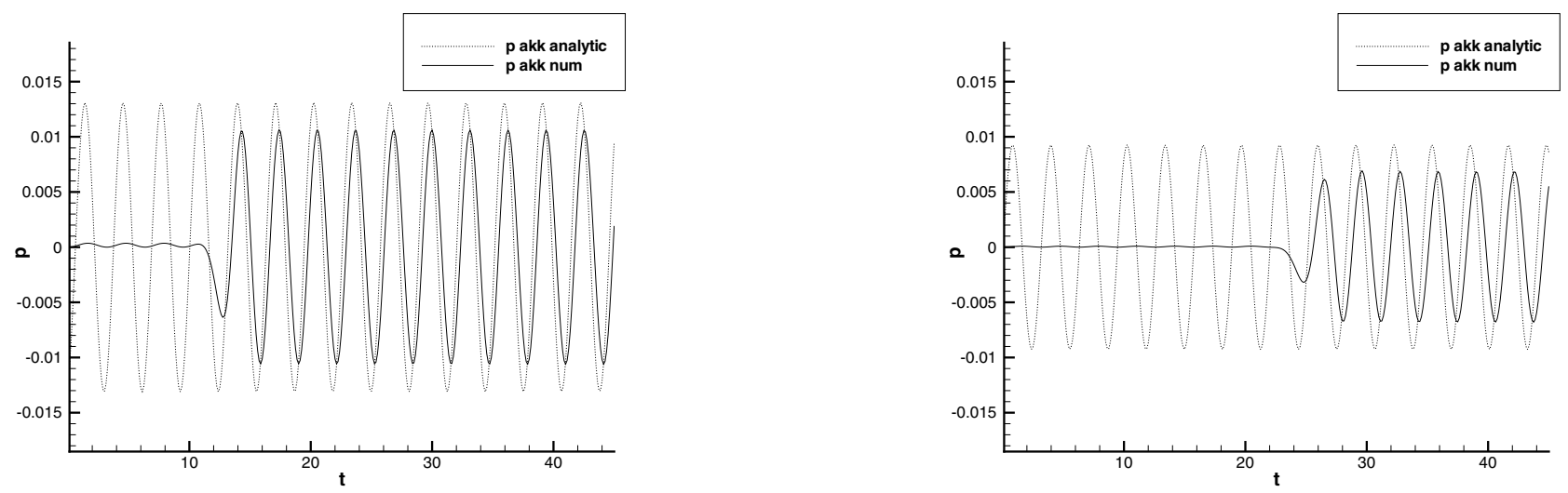

Figure 10. Time plot of the acoustic pressure at point $(150,0)$ and $(300,0), 2$ nd order scheme.

numerical convergence studies have been done up to 24th order of accuracy, in space and time. The twelfth order version in space and time needs as much computational time as the DRP scheme while requiring only $1 / 3$ of the memory and has better wave resolution properties. On unstructured grids, the reconstruction process for very high order schemes gets complicated and expensive. Here, we applied the ADER idea to the Discontinuous Galerkin (DG) framework where piecewise high order polynomial basis functions in each element provide an easy access to all space derivatives which are needed for the Lax-Wendroff procedure [1].

Our philosophy is then to use simultaneously ADER-DG and ADER-FV methods on unstructured as well as on structured Cartesian grids: In the vicinity of complex bodies unstructured grids are used in a small region. They are linked as soon as possible to structured, Cartesian grids. With this domain decomposition it is possible to use high order schemes in practical applications $[16,17]$.

\section{NUMERICAL EXAMPLES AND COMPARISONS}

To compare the three different schemes, a common test case, the so-called rotating quadrupole, is applied to them all. It is a typical test case for aero-acoustic code validation, generated by a pair of two co-rotating vortices of strength $\kappa=\Gamma /(2 \pi)$ each, where $\Gamma$ is the circulation. They are placed at a distance of $2 r_{0}$ and thus each vortex induces a velocity $q=\Gamma /\left(4 \pi r_{0}\right)$ on the other. This causes the vortices to rotate around their common midpoint. Assuming the vortices as point vortices, the exact solution of the potential theory for the incompressible flow field, as well as the exact solution of the acoustic far field equations can be determined analytically for this setting [9]. Therefore, this example is chosen for algorithm validation in 2D.

For the EIF scheme, we took the analytic solution to calculate the incompressible flow and the acoustic source terms in equations (16) exactly. The numerical propagation of the sound waves is then compared to the analytical acoustic solution. We calculated the case with a vortex distance of $r_{0}=1.0$ and a rotating Mach number of $\mathrm{M}=0.095$ which leads to a rotation period of $T=2 \pi$. Since the setting is symmetric, the second half of the period equals the first half, so the seen period is $T=\pi$. The computational domain is set to $[-400,400] \times[-400,400]$ and discretized by $320 \times 320$ grid points, i.e. with grid spacing of $\Delta x=2.5$. Computations were done by discretizing equations (16) with the ADER-scheme. Approximations of second and fourth order in space and time are compared. Figure 9 shows the contour plot of the acoustic pressure. The acoustic source can be seen in the center and acoustic waves that spiral out of the origin.

Figures 10 and 11 compare the results of the numerical simulation to the analytical solution. Two points on the $x$-axes have been chosen at distance $x=150$ and $x=300$ from the origin. At these points, the acoustic pressure is plotted versus time. The end time $t \approx 44$ corresponds to about 7 rotational periods. 

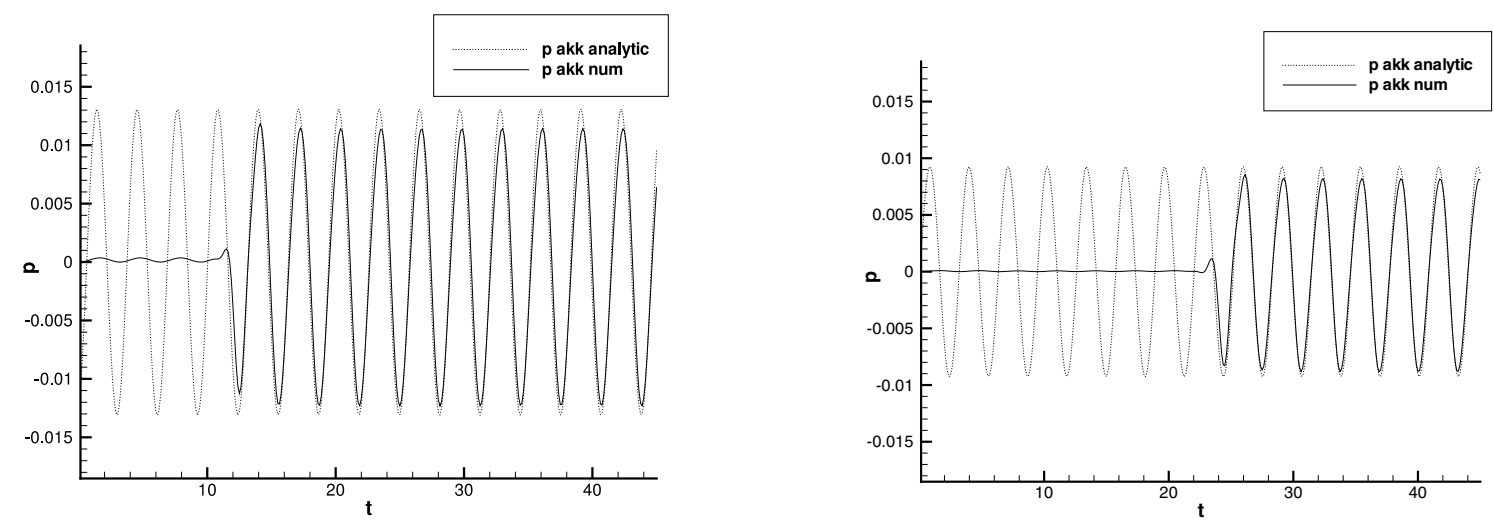

FiguRE 11. Time plot of the acoustic pressure at point $(150,0)$ and $(300,0), 4$ th order scheme.

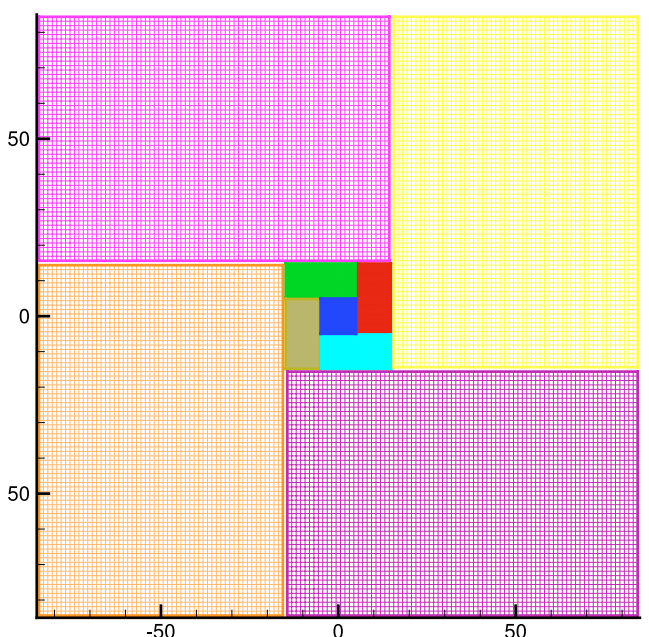

Figure 12. Grid of the ADER scheme with heterogeneous domain decomposition.

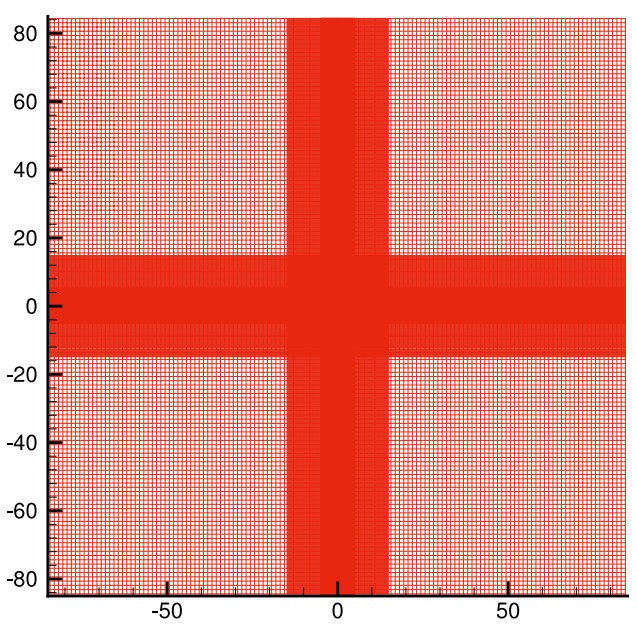

Figure 13. Grid of the MPV scheme: Cartesian non-equidistant.

Figure 10 shows the results of the second order scheme. Since the exact solution is periodic in time and the source terms are calculated from the exact solution, the amplitude remains constant, no damping can be seen over the time. With growing distance from the acoustic source, the amplitude of the numerical wave is damped stronger than the analytical one and a phase error is seen. Using the fourth order scheme for the calculation of the sound waves (Fig. 11), nearly no phase error occurs. The quotient of analytical amplitude to numerical amplitude remains constant with the distance. Only the physical damping of the acoustic wave can be observed. These calculations show the good wave propagation properties of the ADER scheme and emphasize again, that it is necessary for computational aero-acoustics to use high order schemes.

If the incompressible solution is calculated with a numerical scheme, it can be seen that numerical errors in the incompressible solution influence the calculation of the acoustic source terms strongly. Due to the fact that the vortices have an finite extension, they are damped and smeared during their rotation, and the source terms for the acoustic are no longer periodic in time. This case is now considered when comparing the MPV approach and the direct simulation via high order ADER schemes for the non-linear Euler equations. 


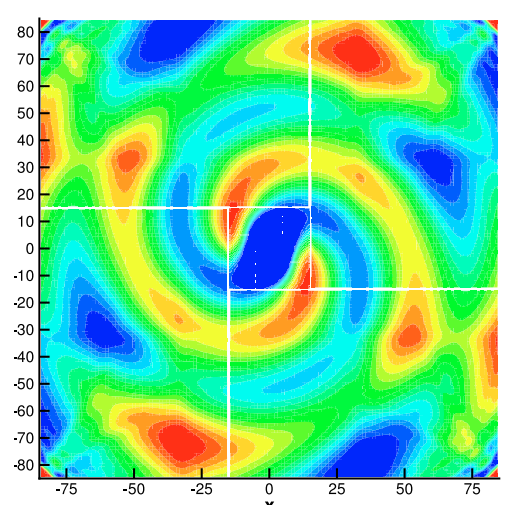

Figure 14. ADER scheme: total pressure $p$.

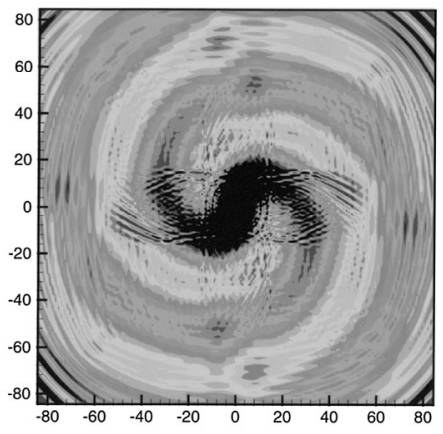

FiguRE 15. MPV scheme: total pressure $p$.

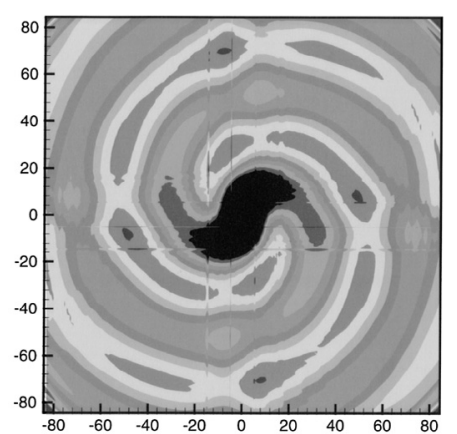

FiguRE 16. MPV scheme: acoustic pressure $p^{(1)}$.
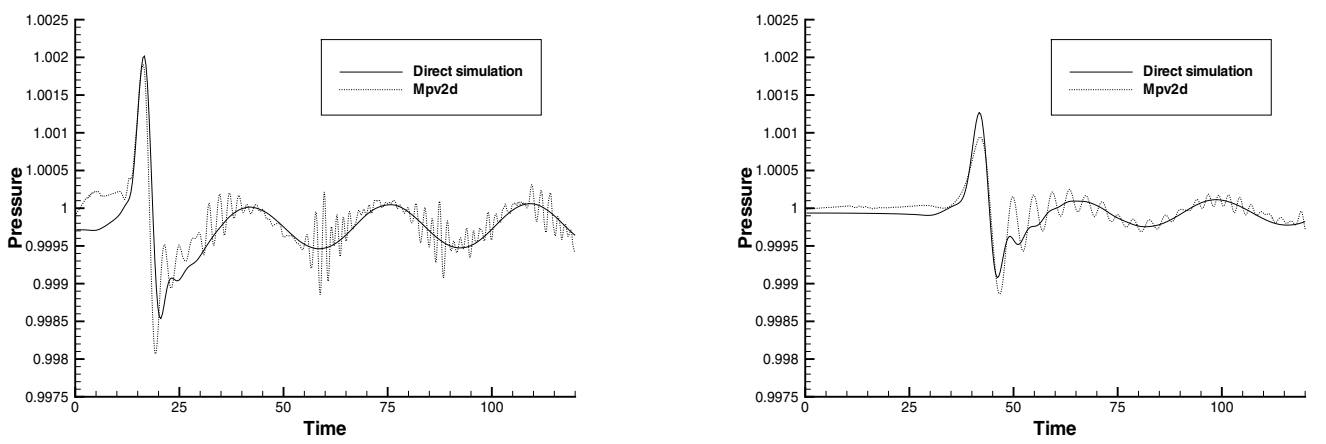

Figure 17. Pressure $p$ obtained with the ADER vs. MPV scheme.

For the comparison of MPV with ADER/heterogeneous domain decomposition, the computational domain was $[-85,85] \times[-85,85]$, discretized by three grids with grid sizes of $\Delta x=0.05,0.3125,1.25$. Figures 12 and 13 show the resulting grids with domain decomposition versus a one-block grid. It is easy to see that the Cartesian non-equidistant grid for the MPV scheme is refined in regions where it is not necessary. This is of coarse not a principle, but an easy to solve implementational problem but it has to be kept in mind when comparing CPU times later on.

Figures 14-16 show the pressure distribution after about 3 seen rotational periods at $t=9.47$. At first, it can be seen that the ADER scheme is able to represent the rotation of the vortices as well as the spreading of the sound waves. The result of the MPV scheme shows a lot more of perturbations. This is due to the strong anisentropy of the grid. The direct simulation used interpolations of ghost cell values at the domain interfaces while the one-block MPV scheme had to cope with it. If the acoustic pressure $p^{(1)}$ is considered which is obtain via large scale averaging for the restriction and calculated on a much coarser and equidistant acoustic grid, the solution is smooth. Besides of these perturbations, the accordance of the two schemes is good.

Again, observation points on the horizontal centerline have been chosen at $x=10.15625$ and $x=50.625$. Figure 17 shows the time plot of the total pressure $p$. Again the solution of the ADER scheme is much smoother than that of the MPV scheme. But besides this, the two solutions are in good accordance with respect to the amplitudes as well as to the phase error. Nearly no phase shift can be seen between the two solutions at the 

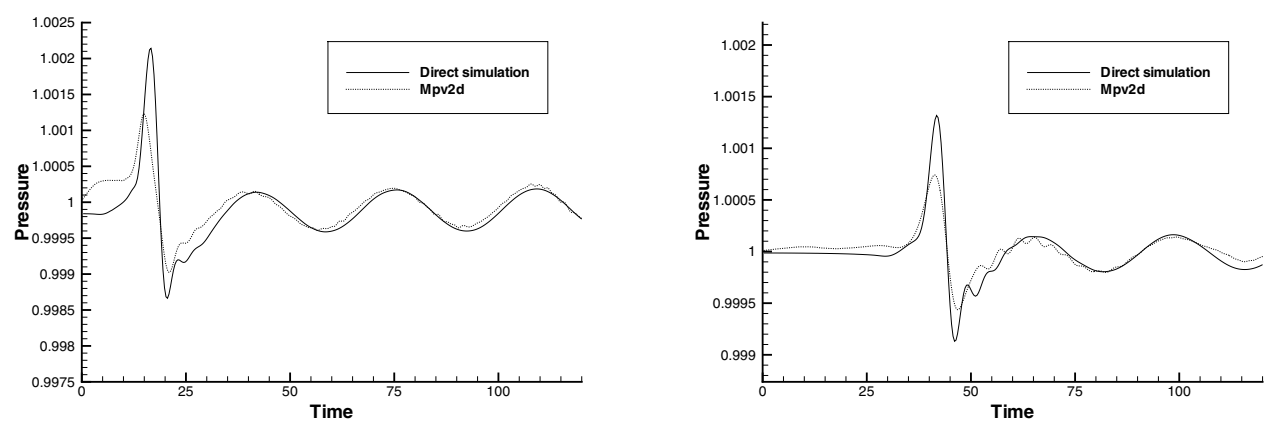

Figure 18. Pressure $p$ obtained with the ADER vs. $p^{(1)}$ obtained with the MPV scheme.

near point as well as on the far point. This emphasizes the good dissipation and dispersion properties of the ADER scheme on the collocated as well as the MPV scheme on the staggered grid arrangement.

Figure 18 shows the total pressure as obtained in the far field from acoustic pressure $p^{(1)}$ plus constant background pressure, i.e. $p=1+\mathrm{M} p^{(1)}$. Here, the comparison between the solution of the two different schemes is again good. Note, that the MPV scheme is a second order scheme while the ADER scheme used fourth order in the innermost and the middle regions of the grid, i.e. in the non-linear Euler regions, and sixth order in the outermost linearized Euler domains.

The comparison of CPU time shows that the EIF scheme is the fastest for this test case which was expected. It took about $5 \mathrm{~min}$ for the acoustic calculation plus the time for the incompressible solution which was about $45 \mathrm{~min}$. The solution with the ADER/heterogeneous domain decomposition method took about 590 min for the 6th order scheme applied here and the second order MPV scheme needed 286 min. Having in mind that the ADER grid contained $200 \times 200+4 * 32 \times 64+4 * 56 \times 112=73280$ grid cells, the MPV grid $376 \times 376=141376$ grid cells, i.e. nearly twice as much and had to apply small time steps even in the outer domains, the ADER calculation is much more expensive than the MPV scheme. The memory requirements of the three schemes were comparable.

\section{Summary}

In this paper we gave an overview over three different classes of schemes that can be applied to aero-acoustical problems. Only one of them, the EIF scheme is especially designed for aero-acoustics. It covers applications where no interactions between flow and acoustics have to be considered because far-field noise is the point of interest. In this case, i.e. if it is applicable at all, this scheme is by far the fastest of the three schemes.

The second class of schemes is the MPV scheme which is designed for low Mach number flows in general, including acoustics, but not only acoustics. It has been shown that this code is able to capture far field noise as well as acoustic-flow interactions as in the baroclinic vortex formation even with a second order approximation. It is also applicable to other low Mach phenomena such as natural convection or other cases with strong thermal gradients which imply strong density changes in an almost incompressible fluid. Thus, the MPV scheme has been shown to cover a wide range of application in all subsonic regimes.

The third class of schemes use the compressible equations directly without any considerations concerning acoustics or low Mach number properties of the flow. It has been shown that this idea in combination with high order approximations is able to give good solutions even in the very low Mach number regime. Using domain decomposition and linearized equations on Cartesian grids as soon as possible reduces the computational effort drastically, so it can be used for practical applications. Nevertheless, acoustic/flow-interaction have to be considered carefully. Applying a scheme of only second order was not sufficient for the simulation of the baroclinic vortex formation, for example. In this case, the use of more than one pressure variable is necessary. 
Since second order schemes are widely used in codes for practical applications, the coupling between the high order domain decomposition and the second order MPV scheme will be further analyzed in the future. Another open point is the parallelization and highly non-trivial load-balancing of this heterogeneous approach.

\section{REFERENCES}

[1] M. Dumbser and C.-D. Munz, Arbitrary High Order Discontinuous Galerkin Schemes. IRMA series in mathematics and theoretical physics.

[2] R. Fortenbach and C.-D. Munz, Multiple Scale considerations for sound generation in low Mach number flow, in Proc. The GAMM Jahrestagung, Augsburg, Germany, March 25-28 (2002).

[3] K. Geratz, Erweiterung eines Godunov-Typ-Verfahrens für zwei-dimensionale kompressible Strömungen auf die Fälle kleiner und verschwindender Machzahl. Ph.D. Thesis, RWTH Aachen (1997).

[4] J. Hardin and D. Pope, An acoustic/viscous splitting technique for computational aeroacoustics. Theoret. Comput. Fluid Dynamics 6 (1994) 323-340.

[5] S. Klainerman and A. Majda, Singular limits of quasilinear hyperbolic systems with large parameters and the incompressible Limit of Compressible Fluids. Comm. Pure Appl. Math. 34 (1981) 481-524.

[6] R. Klein, Semi-implicit extension of a Godunov-type scheme based on low Mach number asymptotics I: One dimensional flow. J. Comput. Phys. 121 (1995) 213-237.

[7] R. Klein, N. Botta, L. Hofmann, A. Meister, C.-D. Munz, S. Roller and T. Sonar, Asymptotic adaptive methods for multiscale problems in fluid mechanics. J. Engrg. Math. 39 (2001) 261-343.

[8] A. Meister, Asymptotic single and multiple scale expansions in the mow Mach number limit. SIAM J. Appl. Math. 60 (1999) $256-271$.

[9] B.E. Mitchell, S.K. Lele and P. Moin, Direct computation of the sound from a compressible co-rotating vortex pair. J. Fluid Mech. 285 (1995) 181-202.

[10] C.-D. Munz, S. Roller, R. Klein and K.J. Geratz, The extension of incompressible flow solvers to the weakly compressible regime. Comput. Fluids 32 (2003) 173-196.

[11] S. Roller, Ein numerisches verfahren zur simulation schwach kompressibler Strömungen. Ph.D. Thesis, University of Stuttgart (2004).

[12] S. Roller and C.-D. Munz, A low Mach number scheme based on multi-scale asymptotics. Comput. Visual. Sci. 3 (2000) 85-91.

[13] T. Schneider, N. Botta, K. Geratz and R. Klein, Extension of finite volume compressible flow solvers to multi-dimensional, variable density zero Mach number flow. J. Comput. Phys. 155 (1999) 248-286.

[14] T. Schwartzkopff, Finite-Volumen Verfahren hoher Ordnung und heterogene Gebietszerlegung für die numerische Aeroakustik. Ph.D. Thesis, University of Stuttgart (2005).

[15] T. Schwartzkopff and C.-D. Munz, Direct simulation of aeroacoustics, in Proc. Applied Mathematics and Mechanics (GAMM 2002) 2 (2002).

[16] T. Schwartzkopff and C.-D. Munz, Direct simulation of aeroacoustics, in Analysis and Simulation of Multifield Problems, W. Wendland and M. Efendiev, Eds., Springer. Lect. Notes Appl. Comput. Mech. 12 (2003) 337-342.

[17] T. Schwartzkopff, M. Dumbser and C.-D. Munz, CAA using domain decomposition and high order methods on structured and unstructured meshes, in 10th AIAA/CEAS Aeroacoustic Conference, Manchester, GB (2004).

[18] T. Schwartzkopff, M. Dumbser and C.-D. Munz, Fast high order ADER schemes for linear hyperbolic equations. J. Comput. Phys. 197 (2004) 532-539.

[19] T. Schwartzkopff, C.-D. Munz, E. Toro and R. Millington, ADER-2d: A very high-order approach for linear hyperbolic systems, in Proceedings of ECCOMAS CFD Conference 2001 (September 2001).

[20] E. Toro and R. Millington, ADER: High-order non-oscillatory advection schemes, in Proceedings of the 8th International Conference on Nonlinear Hyperbolic Problems, preprint (February 2000). 